\title{
Methods for defining distinct bioenergetic profiles in platelets, lymphocytes, monocytes, and neutrophils, and the oxidative burst from human blood
}

\author{
Balu K Chacko*, Philip A Kramer*, Saranya Ravi, Michelle S Johnson, Robert W Hardy, Scott W Ballinger and \\ Victor M Darley-Usmar
}

Peripheral blood mononuclear cells and platelets have long been recognized as having the potential to act as sensitive markers for mitochondrial dysfunction in a broad range of pathological conditions. However, the bioenergetic function of these cells has not been examined from the same donors, yet this is important for the selection of cell types for translational studies. Here, we demonstrate the measurement of cellular bioenergetics in isolated human monocytes, lymphocytes, and platelets, including the oxidative burst from neutrophils and monocytes from individual donors. With the exception of neutrophils, all cell types tested exhibited oxygen consumption that could be ascribed to oxidative phosphorylation with each having a distinct bioenergetic profile and distribution of respiratory chain proteins. In marked contrast, neutrophils were essentially unresponsive to mitochondrial respiratory inhibitors indicating that they have a minimal requirement for oxidative phosphorylation. In monocytes and neutrophils, we demonstrate the stimulation of the oxidative burst using phorbol 12-myristate 13-acetate and its validation in normal human subjects. Taken together, these data suggest that selection of cell type from blood cells is critical for assessing bioenergetic dysfunction and redox biology in translational research.

Laboratory Investigation (2013) 93, 690-700; doi:10.1038/labinvest.2013.53; published online 25 March 2013

KEYWORDS: mitochondria; neutrophils; peripheral blood mononuclear cells; respiratory burst

The analysis of metabolic markers in blood has great utility in research and the clinical laboratory for its pathological and prognostic value in metabolic diseases. For example, a frequently used clinical test for assessing the severity of hyperglycemia in diabetics is the measurement of the glycosylated form of hemoglobin (HbAlc). Experimental translational studies have extended this concept to the measurement of mitochondrial function in diseases such as diabetes based on the concept that systemic exposure to hyperglycemia or pro-inflammatory cytokines impact mitochondria in platelets and monocytes. ${ }^{1-4}$ This is important as key diseases including atherosclerosis, cancer, and neurodegeneration are now known to be associated with deterioration in mitochondrial function. ${ }^{5-9}$ These insights, together with the advent of mitochondrial-targeted drugs, ${ }^{10-12}$ emphasize the need for methods to assess the changing dynamics of bioenergetic function in patient populations. ${ }^{11-13}$ Leukocytes and platelets are the two major cell types in the blood, which have active and detectable oxidative phosphorylation. Several studies in patients with diabetes, cardiovascular, and neurodegenerative diseases have shown significant defects in mitochondrial function in leukocytes and platelets supporting their potential for monitoring the disease process and therapeutic interventions. ${ }^{3,14-18}$

Platelets have frequently been used as a source of material for bioenergetic analysis in translational studies. These anuclear fragments of megakaryocytes have an important role in the regulation of thrombosis and are exposed to systemic metabolic and inflammatory stress during their maturation in the bone marrow. In support of the concept that platelet function can reflect metabolic stress, recent studies have utilized the extracellular flux analyzer to examine the bioenergetics of isolated human platelets. ${ }^{3,14}$ Consistent with the concept that diabetes is associated with mitochondrial dysfunction, lower basal, and maximal respiration rates were found in platelets isolated from type 2 diabetics compared with control subjects. ${ }^{3}$ Interestingly, platelets have been used to identify defects in cytochrome c oxidase in Alzheimer's patients. ${ }^{18}$ The changing function of platelets in ageing has also been studied and it is clear that platelet

Department of Pathology, UAB Mitochondrial Medicine Laboratory, Center for Free Radical Biology, University of Alabama at Birmingham, Birmingham, AL, USA Correspondence: Dr VM Darley-Usmar, PhD, Department of Pathology, UAB Mitochondrial Medicine Laboratory, Center for Free Radical Biology, University of Alabama at Birmingham, Biomedical Research Building II, 901 19th Street South, Birmingham, AL 35294, USA.

E-mail: darley@uab.edu

*These authors contributed equally to this work.

Received 7 December 2012; revised 15 February 2013; accepted 24 February 2013 
mitochondrial function decreases with ageing, partly owing to a loss of complex I activity. ${ }^{19-21}$

Bioenergetics in monocytes have also been studied in human subjects and it is now becoming clear that mitochondria have a critical role in the phenotype switching of macrophages. The balance between the pro-inflammatory M1 and anti-inflammatory M2 phenotypes of peripheral blood monocytes has been suggested to be one of the potential mechanisms leading to metabolic syndrome and arterial stiffness in obese type 2 diabetic patients. ${ }^{22}$ Recent reports have shown that altered monocyte mitochondrial respiration is correlated with mortality following septic shock. ${ }^{16}$ Studies in human mononuclear cells isolated from type 2 diabetic patients suggest a correlation of perturbations of mitochondrial homeostasis to alterations in mitochondrial morphology, mitochondrial mass, and membrane potential. ${ }^{17} \mathrm{~A}$ recent study in fibromyalgia patients demonstrated that increased lipid peroxidation, mitochondrial dysfunction, and mitophagy in blood mononuclear cells are strongly associated with clinical symptoms of the disease. ${ }^{15}$ It is also now clear that both T and $\mathrm{B}$ cells modulate their metabolism during normal function, and mitochondrial bioenergetics also has a key role in this process. ${ }^{23,24}$ Among the likely mediators resulting in bioenergetic dysfunction in leukocytes and platelets are cytokines. Importantly, environmental, dietary, and epidemiological studies demonstrate distinct patterns of cytokine profiles in patients with chronic inflammatory diseases, such as cancer, diabetes, obesity, and metabolic syndrome. ${ }^{25-28}$ These data highlight how measurement of cellular bioenergetics in leukocytes and platelets can act as a surrogate index of mitochondrial function in several pathologies including diabetes and Alzheimer's disease or in some cases can be directly related to the underlying pathology such as the autoimmune diseases. ${ }^{18,29}$

In addition to the mitochondrial consumption of oxygen at cytochrome $c$ oxidase, the NADPH oxidases are also capable of mitochondrial-independent oxygen consumption. Chronic inflammatory pathologies cause priming of neutrophils and monocytes, which results in enhanced production of superoxide from NADPH oxidase (NOX 2) through the oxidative burst. ${ }^{30-33}$ The levels of activation of the oxidative burst are thought to be a sensitive index of autoimmune conditions, and oxygen consumption can reach levels that are comparable if not greater than mitochondrial respiration. Indeed, recent studies have demonstrated an inverse association of oxidative burst capacity and disease severity in the recurrence of Guillian Barre' syndrome, arthritis, Crone's disease, multiple sclerosis, and other autoimmune conditions in both human subjects and animal models. ${ }^{34-36} \mathrm{~A}$ few case reports have also been described of patients with G6PD deficiency and the resulting recurrent infections associated with decreased NADPH for the oxidative burst in neutrophils. ${ }^{37,38}$ Defects in the neutrophil oxidative burst results in recurrent infections, the formation of granulomas, and other immune-deficient pathologies. ${ }^{39}$ Clinical testing for deficiencies in the oxidative burst currently use the qualitative and nonspecific reduction of nitroblue tetrazolium. ${ }^{40,41}$

It is clear that a high throughput assay for the sensitive measurement of oxygen consumption in cells isolated from $10-20 \mathrm{ml}$ of human blood could give insights into both cellular bioenergetics in a broad range of diseases and innate immunity, and then be beneficial for translational research. In the present study, we demonstrate how extracellular flux analysis can define a bioenergetic profile in platelets, monocytes, lymphocytes, and measurement of the oxidative burst in monocytes and neutrophils from individual donors. A comparison of the bioenergetic profiles of these cell types from single individuals has not been previously reported. Interestingly, control of the respiratory chain appears to be quite different between platelets, monocytes, and lymphocytes, and this should be taken into account in the design of translational studies.

\section{MATERIALS AND METHODS Blood Collection and Cell Isolations}

All study protocols for collection and handling of human samples were reviewed and approved by the Institutional Review Board, University of Alabama at Birmingham. Blood samples (1-2 tubes, $8.5 \mathrm{ml} /$ tube) were collected from eight healthy volunteers (five males and three females, 25-55 years of age) in vacutainers (BD Biosciences) containing $1.5 \mathrm{ml}$ ACD solution (trisodium citrate, $22.0 \mathrm{~g} / \mathrm{l}$; citric acid, $8.0 \mathrm{~g} / \mathrm{l}$; and dextrose $24.5 \mathrm{~g} / \mathrm{l}$ ) as anticoagulant and processed within $15 \mathrm{~min}$ of collection. All isolation procedures were designed to prevent activation of the cells during isolation such as performing isolations at room temperature with the use of anticoagulants. We found that the frequently recommended use of phosphate-buffered saline to prepare leukocytes resulted in severe loss of bioenergetic function and for this reason we use RPMI Cat \#17-105-CV (no antibiotics, no phenol red, no FBS). The artifactual activation of cells during the preparation results in clumping, and if this occurs the samples should be discarded. Platelet-rich plasma (PRP) and the buffy coat were separated by centrifugation at $500 \mathrm{~g}$ for $10 \mathrm{~min}$. Platelets were pelleted by centrifugation of the PRP at $1500 \mathrm{~g}$ for $10 \mathrm{~min}$ and then washed once with PBS containing prostaglandin $\mathrm{I}_{2}\left(\mathrm{PGI}_{2} 1 \mu \mathrm{g} / \mathrm{ml}\right)$ to inhibit aggregation. If $\mathrm{PGI}_{2}$ is not included, the platelets irreversibly aggregate and cannot be purified further. Platelet counts were determined by turbidimetry as reported by Walkowiak et al. ${ }^{42}$ The buffy coat was diluted (1:4) with basal RPMI media and applied to a Histopaque density gradient (specific gravity 1.077, Sigma Chemical Cat \#10771 and 1.119, Cat \#11191), and centrifuged without application of the brake at $700 \mathrm{~g}$ for $30 \mathrm{~min}$. The peripheral blood mononuclear cells (PBMCs) and polymorphonuclear cells (granulocytes) were collected separately. $\mathrm{CD}_{14}{ }^{+}$monocytes were purified from PBMC fraction by the MACS technique (Milteneyi Biotec) using 
superparamagnetic iron-dextran microbead-labeled antiCD14 antibodies according to manufacturer's instructions. PBMC were incubated with the labeled anti-CD14 antibodies at $4{ }^{\circ} \mathrm{C}$ for $15 \mathrm{~min}$ before applying the cells to the column placed in the magnetic field. Cells retained in the column were collected by eluting with RPMI containing $0.5 \%$ bovine serum albumin after removing from the magnetic field. The flow through from the $\mathrm{CD} 14^{+}$monocyte isolation contained lymphocytes, which were further purified by selectively depleting the contaminating platelets and red blood cell (RBC) using magnetic beads labeled with anti-CD65 antibody and anti-CD235a (glycophorin) antibodies, respectively. The purity of each fraction was assessed using FACS analysis and is reported in Supplementary Table 1, and fluorescently conjugated antibodies were used to identify each cell type, monocytes (FITC anti-CD14), lymphocytes (APC anti$\mathrm{CD} 45$ ), and neutrophils (PE anti-CD15). With the exception of monocytes, which were contaminated with $\sim 11 \%$ lymphocytes and $35 \%$ platelets by cell number, all other cell types were over $80 \%$ pure. RBCs are minor contaminants of the neutrophil, lymphocyte, and platelet preparations but do not contribute to bioenergetic measurements. It is important to note that platelet volumes are $\sim 10 \%$ of the volume of monocytes, and we estimate in Supplementary Table 1 that their contribution to the cellular bioenergetics and total protein of the monocyte preparation to be $<1 \%$. On this basis, we conclude that the bioenergetics and protein analysis of the specific isolated cell population have a minimal contribution from contaminating cell types.

As the cell types examined in this study could be activated by the preparation they were examined by microscopy with and without activation by phorbol 12-myristate 13-acetate (PMA) as a positive control. As shown in Supplementary Figure 1, platelets, lymphocytes, neutrophils or monocytes showed no evidence of activation when compared with the positive control. All cells exhibited $>95 \%$ cell viability assessed using trypan Blue exclusion (data not shown).

\section{Attachment of Cells to the Assay Plates for Bioenergetic Assessment}

Determination of cellular bioenergetics and oxidative burst were performed after plating the cells on 24-well polystyrene plates designed for the extracellular flux analyzer. Purified lymphocytes, monocytes, and neutrophils were resuspended in sterile XF assay buffer (DMEM supplemented with $5.5 \mathrm{mM}$ D-glucose, $4 \mathrm{mM}$ L-glutamine, and $1 \mathrm{mM}$ pyruvate, $\mathrm{pH} 7.4$ ), and plated (250 000 cells/well) in $200 \mu \mathrm{l}$ on the CellTak (BD Biosciences)-coated assay plates, and allowed to settle for $30 \mathrm{~min}$ at $37^{\circ} \mathrm{C}$. The sedimented cells were attached to the bottom of the plate by centrifugation at $40 \mathrm{~g}$ without application of the brake and then brought up to $660 \mu \mathrm{l}$ using XF assay medium. Platelets were plated at 25 million per well for bioenergetic measurements after removing $\mathrm{PGI}_{2}$ by washing. The plating efficiency was calculated by counting the cells on the plate and assuming that the seeding number represented
$100 \%$. On this basis, the plating efficiencies for all cell types were $>70 \%$ (monocytes, $76.0 \pm 2.7 \%$; lymphocytes, $74.8 \pm$ $14.0 \%$; and neutrophils, $70.2 \pm 2.7$ ) for four independent subjects, which suggests that the subsequent measurements are representative of the population of cells isolated from each individual.

\section{Measurement of Cellular Bioenergetics}

Cellular bioenergetics of the isolated cells was determined using the extracellular flux analyzer (Seahorse Bioscience), which measures $\mathrm{O}_{2}$ and protons. This system allows for realtime, noninvasive measurements of $\mathrm{O}_{2}$ consumption rate (OCR) and proton production rate (PPR), which can be correlated to mitochondrial function/oxidative burst and glycolysis, respectively. ${ }^{43}$ The injection ports attached to the wells allow for injection of inhibitors of mitochondrial respiratory chain or activators of the oxidative burst to determine the defects in individual cellular respiration pathways or enzymes. Pilot experiments for monocytes, neutrophils, platelets, and lymphocytes isolated from individual donors were performed to determine the optimal cell number required for accurate measurements of OCR and PPR. The optimum concentration of the inhibitors and activators to be used for the assessment of mitochondrial function and oxidative burst were determined by titrating the individual compounds in separate experiments against the cell number determined in the first set of experiments. First, the mean basal respiration is determined by taking 3-4 OCR measurements before the addition of the inhibitors or activators. ATP-linked OCR and proton leak were determined by injecting oligomycin at $0.5 \mu \mathrm{M}$ (for monocytes, lymphocytes, and neutrophils) or $0.75 \mu \mathrm{M}$ (for platelets). The fall in OCR following oligomycin injection is the rate of oxygen consumption that corresponds to ATP synthesis, and the oligomycin-insensitive rate is considered as proton leak across the inner mitochondrial membrane. FCCP, an uncoupler of the electron transport chain, was used at a concentration of $0.6 \mu \mathrm{M}$ to determine the maximal respiration rate. This rate gives the theoretical maximum oxygen consumption that can take place at cytochrome c oxidase whether limited by availability of substrate or activity of the electron transport chain. The difference between the basal rate and this FCCPstimulated rate is the reserve capacity of the mitochondrion, which is a measure of the maximal potential respiratory capacity the cell can utilize under conditions of stress and/or increased energetic demands. Antimycin A, an inhibitor of Complex III, was used to completely inhibit mitochondrial electron transport. The OCR determined after antimycin A injection is attributable to non-mitochondrial oxygen consumption. Mitochondrial basal respiration, proton leak, and the maximal respiration were calculated after correcting for the non-mitochondrial OCR for each assay. Cells were allowed to attach to the XF24 plate for 30-60 min before measurement of mitochondrial function. Under these conditions, viability was over $90 \%$ for all cell types and remained 
so over the time course of the assay. At the end of the assay period, cell lysates were collected, and OCR and PPR values normalized to the protein content in each well.

\section{Measurement of the Oxidative Burst}

Determination of oxidative burst was performed in the same cells with or without determination of mitochondrial function as described above using the extracellular flux analyzer. Cells were activated with PMA $(100 \mathrm{ng} / \mathrm{ml})$, a well characterized activator of protein kinase $\mathrm{C}$ (PKC) and inducer of oxidative burst. The difference between the PMA-stimulated OCR and the rate of oxygen consumption after antimycin A treatment was taken as the oxygen consumption owing to oxidative burst. To compare the production of superoxide from neutrophils with an established method, the reduction of cytochrome $c$ was used. ${ }^{44}$ Briefly, $2 \times 10^{5} \mathrm{CD}^{+} 5^{+}$ neutrophils were incubated in the presence of $20 \mu \mathrm{m}$ horse heart cytochrome $c$ (Sigma Chemical) and monitored for the SOD-inhibitable reduction of cytochrome $c$ before and after the addition of PMA by spectrophotometry. Concentrations of reduced cytochrome $\mathrm{c}$ were measured using the extinction coefficient $18.14 / \mathrm{mM} / \mathrm{cm}$ (at $550-556 \mathrm{~nm}$ ). Superoxide generation following PMA activation of neutrophils was also determined using the semiquantitative nitroblutetrazolium reduction assay and assessment of the formazan product in activated neutrophils. ${ }^{41}$

Determination of Mitochondrial Respiratory Complexes Mitochondrial respiratory chain complexes relative to the total protein in each cell type were determined by western blotting for cytochrome $c$ oxidase subunit I and Reiske ironsulfur protein, two mitochondrial proteins required for electron transport. After isolating the different cell types from the blood, samples were lysed and the proteins solubilized by mixing with $150 \mu \mathrm{l}$ lysis buffer $(50 \mathrm{mM}$ Tris- $\mathrm{HCl} \mathrm{pH}$ 7.4, $150 \mathrm{mM} \mathrm{NaCl}, 0.5 \%$ sodium deoxycholate, $0.1 \%$ SDS, $1 \mathrm{mM}$ EDTA, and $1 \%$ NP-40) containing $1 \times$ protease inhibitor cocktail (Cat \# 14150700, Roche, USA) and then performing a freeze-thaw cycle. The soluble fraction of the cell lysate was collected by centrifuging at $10000 \mathrm{~g}$ for $10 \mathrm{~min}$. For western blot analysis, $50 \mu \mathrm{g}$ protein was loaded per well and probed using anti-cytochrome c oxidase subunit I (Cat \# A6403, Invitrogen, USA) and anti-Reiske iron-sulfur protein antibody (Cat \# A21346, Invitrogen). Densitometric analysis was performed using AlphaView SA software (Alpha Innotech, CA, USA) to determine the relative levels of these proteins. Data were normalized to the total signal from the western blot membrane stained with Ponceau and expressed as arbitrary units.

\section{Assessement of Purity of the Isolated Fractions by FACS Analysis}

The purity of isolated cell fractions, monocytes, lymphocytes, and granulocytes, were determined by FACS analysis after staining the cells using anti-CD14-FITC, anti-CD45 PE, and
anti-CD15-APC antibodies, respectively. Platelet purity was determined using forward/side scattering method.

\section{Statistics}

Each blood sample was analyzed with 3-5 replicates for bioenergetic determinations, and data are presented as mean \pm s.e.m. Statistical significance was determined using Student's $t$-test, with $P<0.05$ taken as significantly different.

\section{RESULTS}

\section{Cellular Bioenergetics in Monocytes, Platelets, Lymphocytes, and Neutrophils}

Shown in Figure 1 are typical bioenergetic profiles for monocytes, lymphocytes, platelets, and neutrophils from individual donors. Mitochondrial bioenergetic profiles were determined using the sequential addition of inhibitors of oxidative phosphorylation as described previously. ${ }^{43}$ After obtaining a basal OCR, oligomycin addition inhibited ATP synthesis, resulting in a decrease in OCR for all cell types except neutrophils (Figure 1). Next, the proton ionophore FCCP was added to cells that resulted in significant stimulation of OCR in monocytes and lymphocytes but had little effect on platelets or neutrophils. Finally, the complex III inhibitor antimycin A was injected to determine the nonmitochondrial OCR (Figure 1). Interestingly, neutrophils showed little or no response to mitochondrial inhibitors, consistent with a minimal role of oxidative phosphorylation in these cells.

Shown in Figure 2 are the cumulative bioenergetic profiles for 6-8 healthy subjects for monocytes, lymphocytes, and platelets. All these cell types exhibited a detectable basal OCR that ranged from $1.5-2.0 \mathrm{pmolO}_{2} / \mathrm{min} / \mu$ g protein (Figure 2). The amount of basal OCR dedicated for ATP production is the difference between basal and oligomycin-induced OCRs (ATP-linked, Figure 1). ATP-linked OCR was greatest in the platelets with the lymphocytes possessing the lowest values. The OCR remaining after oligomycin addition represents a measure of proton leak (after correction for non-mitochondrial OCR). Consistent with the ATP-linked OCRs, platelets, and lymphocytes showed the lowest and highest values in this parameter, respectively. Maximal OCRs (obtained by adding FCCP) were similar for platelets, lymphocytes, and monocytes. Reserve capacity (the difference between the basal and maximal OCR, a reflection of the maximal mitochondrial electron transport ${ }^{45}$ ) was greatest in the monocytes and significantly less in the platelets (Figure 2). Also shown in Figure $2 \mathrm{~g}$ are pie charts depicting the proportional distribution of OCR among the different categories defined in the bioenergetic profile. From this analysis, it is evident that platelets have the highest ATP-linked respiration and lowest reserve capacity of the cell types examined.

Under the same conditions shown in Figure 1, the basal PPR was measured for all cell types and is shown plotted against the basal OCR with non-mitochondrial OCR subtracted (Figure 3). PPR includes contributions from several 

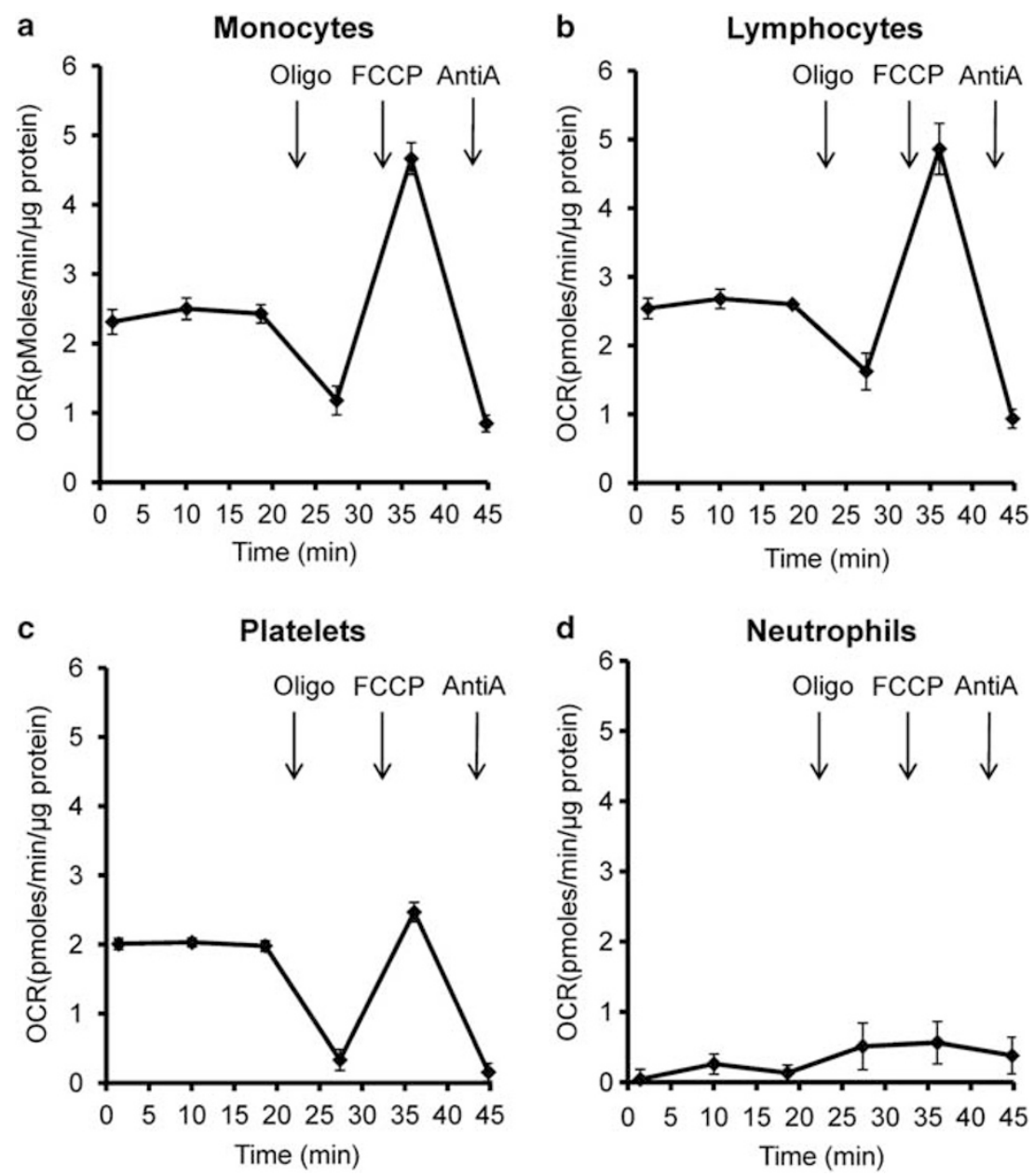

Figure 1 The bioenergetic profiles of human leukocytes and platelets. The bioenergetic profiles of monocytes (a), lymphocytes (b), platelets (c), and neutrophils (d) were determined using the extracellular flux analyzer. The basal measurements of oxygen consumption rates (OCR) were followed by sequential injections of $0.5 \mu \mathrm{g} / \mathrm{ml}$ oligomycin $(0.75 \mu \mathrm{g} / \mathrm{ml}$ for platelets), $0.6 \mu \mathrm{M} \mathrm{FCCP}$, and $10 \mu \mathrm{M}$ antimycin A to determine the indices of mitochondrial function. Data expressed as mean \pm s.e.m. from representative donor profiles, $n=3-5$ assay replicates per sample.

metabolic pathways but under these conditions we assume the major contributor is glycolysis. ${ }^{43}$ From these data, it is clear that each cell type utilizes glycolysis and oxidative phosphorylation to satisfy their metabolic requirements in a distinct fashion (Figure 3a). Monocytes are the most energetic cell type at their basal state with high levels of both glycolysis and oxidative phosphorylation. Lymphocytes and platelets are more oxidative and less glycolytic under basal conditions whereas neutrophils show a moderate PPR with essentially little or no mitochondrial OCR.

Figure $3 b$ shows the fold change in PPR after the addition of oligomycin, which stimulates glycolysis due to the loss of mitochondrial ATP production. Under these conditions, platelets had an $\sim 3$-fold increase in PPR, suggesting that they have substantial glycolytic capacity. Monocytes and neutrophils both showed little or no increase after oligomycin, suggesting that they may be operating at near their maximum glycolytic capacity at basal conditions. Although lymphocytes showed an $\sim 2$-fold increase post oligomycin, they exhibited the lowest absolute value for PPR (Figure 3a).

The differential metabolic profiles for platelets, monocytes, and lymphocytes suggested us that monocytes and lymphocytes could have greater levels of electron transport proteins relative to platelets. To test this, we measured levels of cytochrome c oxidase subunit I and the Rieske Iron sulfur protein (RIS) in complex III using western blotting in the three cell types isolated from three individual donors (Figure 4). The levels of cytochrome $c$ oxidase were highest in monocytes and lymphocytes. Interestingly, the levels of RIS were different between all cell types with the highest levels found in lymphocytes. These mitochondrial proteins could not be detected in neutrophils (result not shown).

\section{Oxidative Burst in Neutrophils and Monocytes}

Activation of phagocytic cells induces a rapid consumption of oxygen by NADPH oxidase-2 (NOX-2 gp91 ${ }^{\text {phox }}$ ) to form 

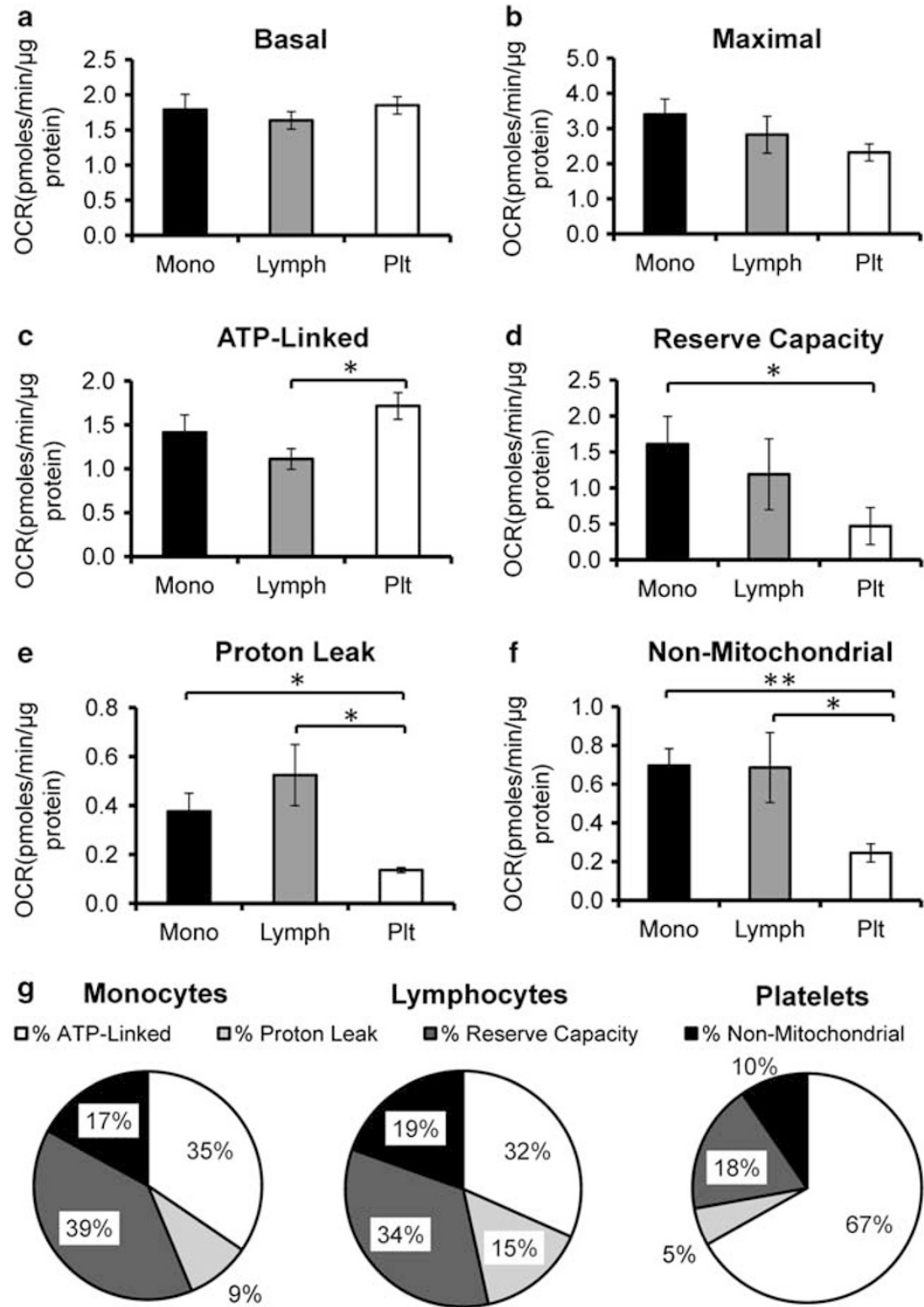

Lymphocytes

ם\% Reserve Capacity
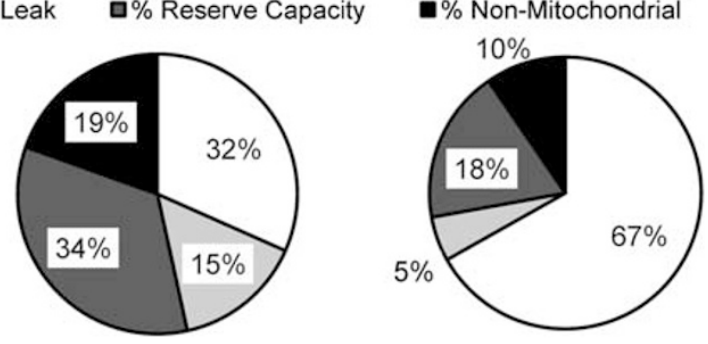

Figure 2 Indices of mitochondrial respiratory function from monocytes, lymphocytes, and platelets. The critical indices of mitochondrial function were calculated from the cellular bioenergetic profiles for each cell type. Basal (basal rate-antimycin A rate) (a), maximal (FCCP rate-antimycin A rate) (b), ATP-linked (basal rate-oligomycin rate) (c), reserve capacity (FCCP response-basal rate) (d), proton leak (oligomycin response-antimycin A rate) (e), and non-mitochondrial (f) OCR of monocytes, lymphocytes, and platelets were plotted. Comparison of the percentage oxygen consumption (g) demonstrates distinct oxygen utilization pattern of each cell type. Cumulative data from eight healthy donors expressed as mean \pm s.e.m. ${ }^{*} P \leq 0.05$, $* * 0.005$

superoxide, which is frequently known as the oxidative burst. This parameter has been widely used as a marker of the activation of innate immunity in human subjects. To determine if this can be detected and quantified in the XF24 analyzer, the OCR of neutrophils was measured at baseline and then after addition of PMA, a well-defined activator of PKC and subsequently the oxidative burst. In Figure 5a, neutrophils show a low basal OCR, which was stimulated $\sim 250$-fold by PMA $(100 \mathrm{ng} / \mathrm{ml})$; this effect was completely inhibited by $10 \mu \mathrm{M}$ diphenylene iodonium (DPI), a flavoprotein inhibitor
(Figures $5 \mathrm{a}$ and $\mathrm{b}$ ). Next, the effects of PMA on monocytes were examined, and similar effects were observed in that addition of PMA significantly increased (6-7-fold) OCR (Figures $5 \mathrm{c}$ and d). As DPI can also inhibit mitochondrial respiration, basal OCR in monocytes was slightly decreased with the addition of DPI, but the PMA-stimulated OCR was almost completely inhibited. The PPR was also stimulated on addition of PMA to $\sim 50 \%$ of the value for OCR and was substantially inhibited by DPI (data not shown). These results are consistent with the oxidation of NADPH forming 

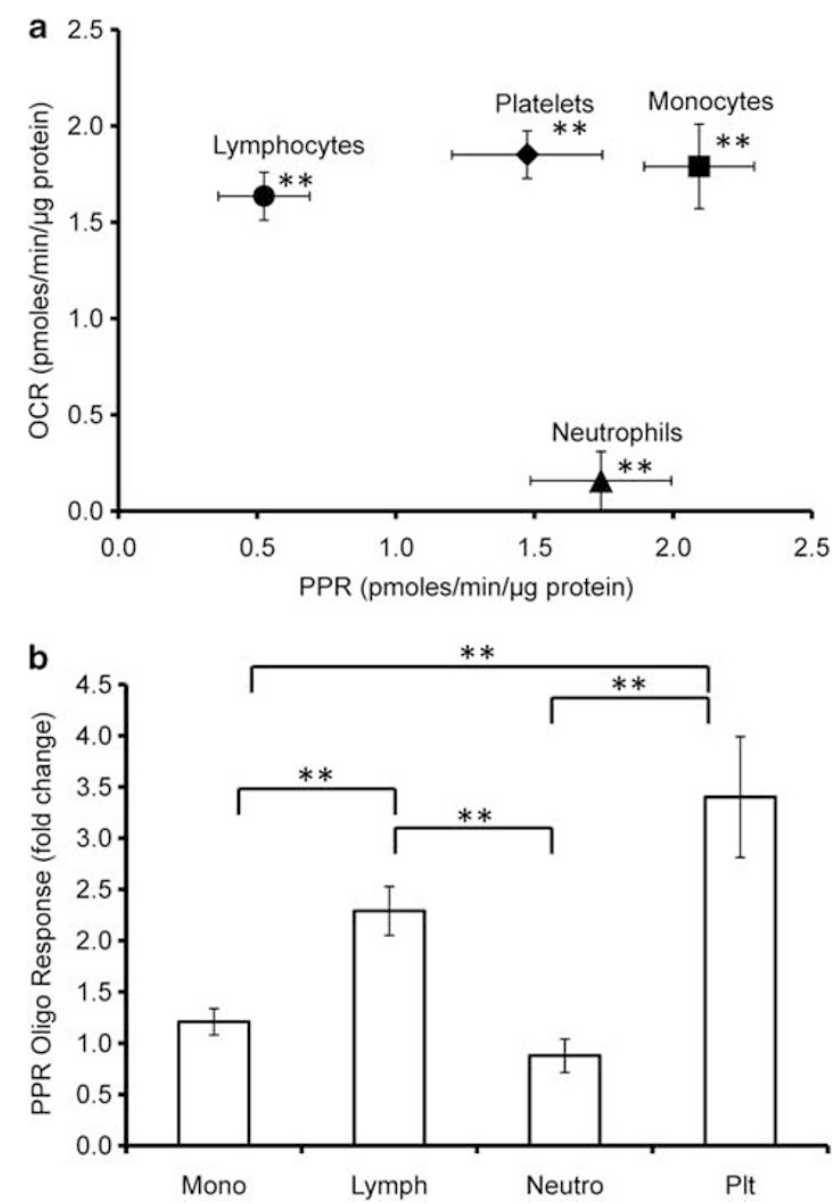

Figure 3 The oxygen consumption rate (OCR) and PPR of each cell type. (a) The mean basal OCR vs basal PPR. (b) The fold increase in PPR after treatment with $0.5 \mu \mathrm{g} / \mathrm{ml}$ oligomycin $(0.75 \mu \mathrm{g} / \mathrm{ml}$ for platelets). Cumulative data from eight healthy donors expressed as mean \pm s.e.m. ${ }^{* *} P \leq 0.005$.

two superoxide molecules (measured as increase in OCR) and one proton (measured as increase in PPR), and therefore suggest that PPR under these conditions can largely be ascribed to the activity of NOX2. Using the same conditions and the cytochrome $c$ reduction assay for the measurement of superoxide, ${ }^{44}$ the rate of PMA-dependent and superoxide dismutase-inhibitable superoxide production was found to be $58.4 \pm 2.0 \mathrm{pmol} / 10000$ cells, which compares favorably with the value of $38.1 \times 2.9 \mathrm{pmol} / 10000$ cells (mean \pm s.e.m., $n=3-5)$ determined with the same cells in the XF24. The production of superoxide by activated neutrophils was also confirmed in a parallel plate using the nitrobluetetrazolium assay, which is used clinically as a screen for NADPH oxidase activity (Supplementary data; Figure 2). Hence, overall, these data provide a new means for quantifying NADPH oxidaserelated oxidative burst in human subjects, which can be used as a surrogate marker for the effects of modulators of the innate immune system in real time.

To determine if the oxidative burst could be measured in monocytes or neutrophils after performing the bioenergetic profile experiments, PMA was injected after antimycin A and
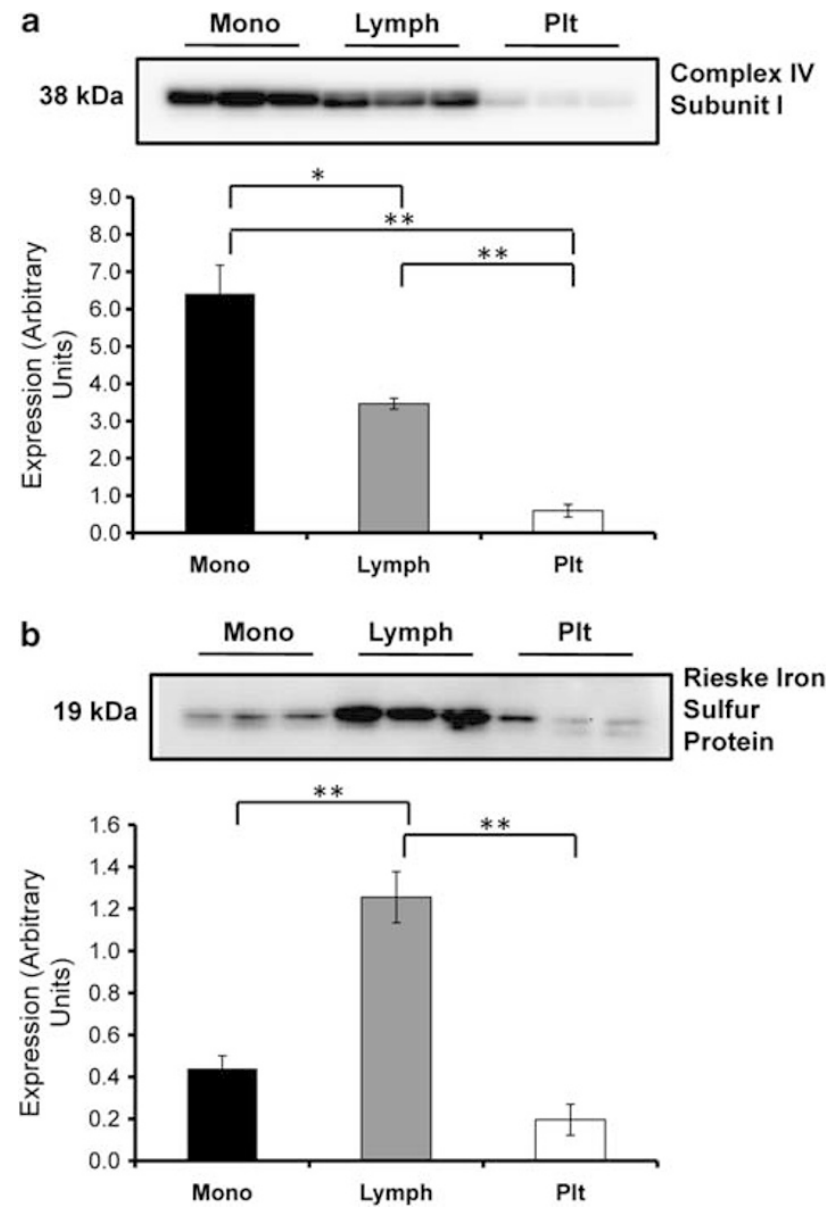

Figure 4 Levels of electron transport chain proteins in peripheral blood cells isolated from freshly drawn human blood. Monocytes, lymphocytes, and platelets isolated from blood were lysed with RIPA buffer, and $50 \mu \mathrm{g}$ of protein was separated on a SDS-PAGE gel followed by western blot for (a) Complex IV subunit I and (b) RIS protein. Protein expression was quantified using AlphaView SA software. Data presented as mean \pm s.e.m., $n=3$. ${ }^{*} P \leq 0.05,{ }^{*} P \leq 0.005$.

compared with cells treated as described in Figure 5. No significant differences in the oxidative burst capacity were observed indicating that the oxidative burst can be measured independently of mitochondrial respiration in the same cells (Figure 6). Figure $7 \mathrm{a}$ shows the cumulative data of 6-8 subjects for the PMA-dependent OCR after measurement of the bioenergetic profile. As expected, neither lymphocytes nor platelets showed any stimulation of OCR on addition of PMA. As mentioned above, the PPR response to PMA also correlates with OCR in each cell type and suggests that $\mathrm{NADPH}$ oxidase activity is responsible for this response (Figure $7 b$ ).

\section{DISCUSSION}

A minimally invasive and rapid protocol for measurement of cellular bioenergetics has great potential for providing important clinical information in certain disease settings. This assay holds the potential to identify those individuals at risk 
a

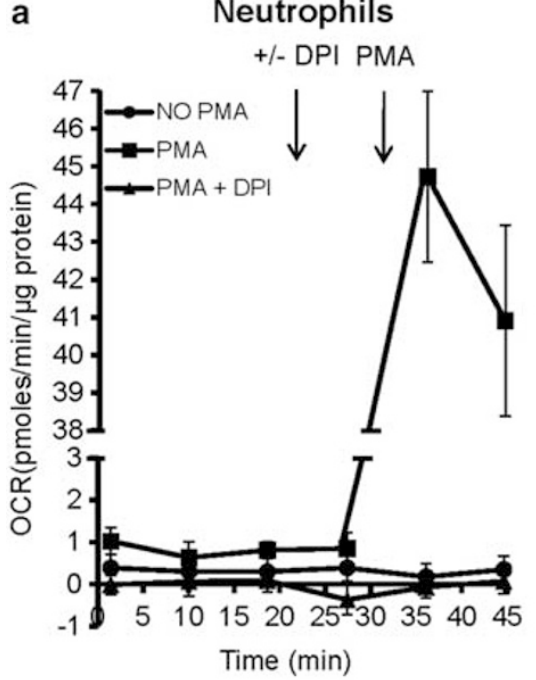

c
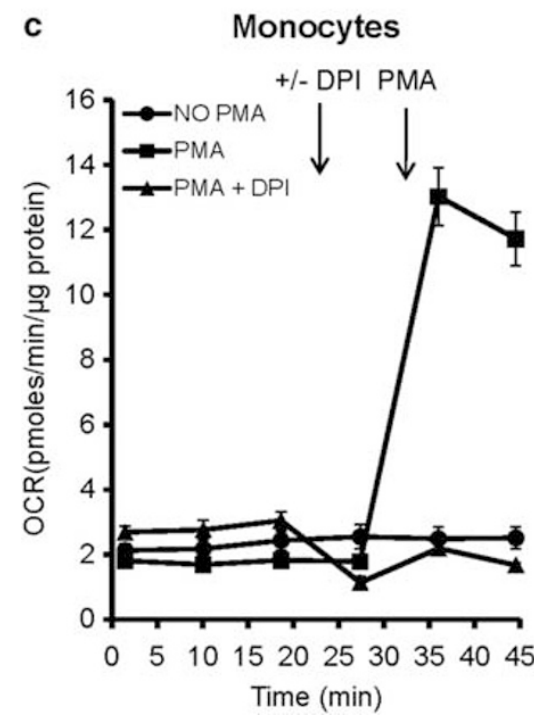

b

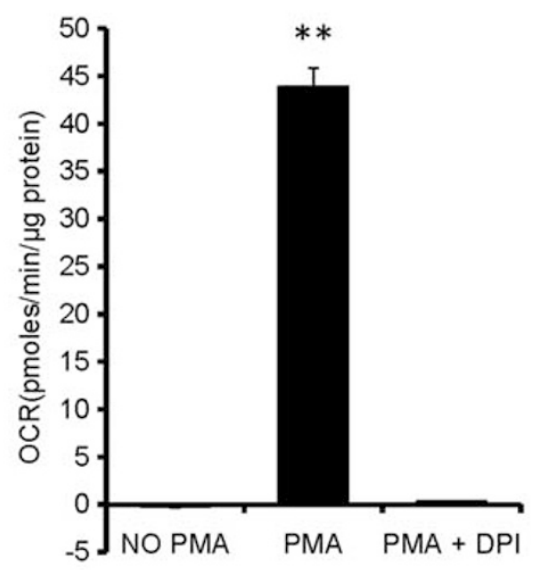

d

Monocytes

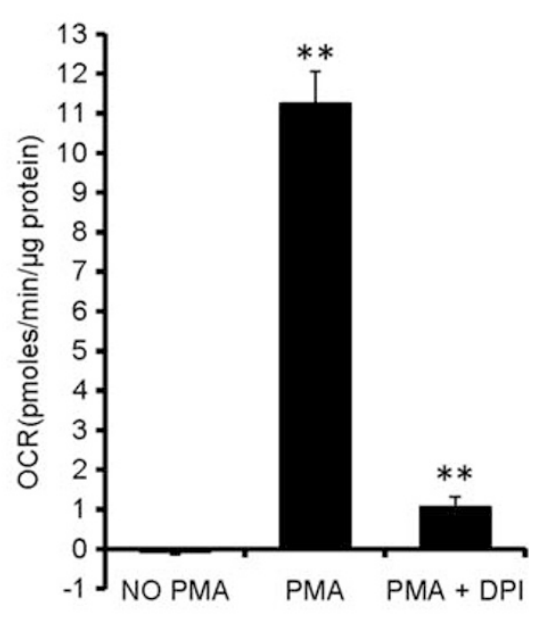

Figure 5 Activating the oxidative burst in monocytes and neutrophils. (a) The OCR of CD15 ${ }^{+}$neutrophils before and after injection of $100 \mathrm{ng} / \mathrm{ml}$ $\mathrm{PMA} \pm 10 \mu \mathrm{M}$ DPI, PMA-dependent response quantified in (b). (c) CD14 ${ }^{+}$monocyte OCR before and after injection of $100 \mathrm{ng} / \mathrm{ml} \mathrm{PMA} \pm 10 \mu \mathrm{M}$ DPI, PMAdependent response quantified in (d). The mean values from a single donor \pm s.e.m. $n=3-5$ assay replicates per sample. ${ }^{* *} P \leq 0.005$.

for rapid disease progression and will provide the benefit of personalized treatment regimens as well as monitoring the effectiveness of treatment and contributing to prognoses. For the analysis of cellular bioenergetics and the oxidative burst to be used in a clinical setting, the techniques described here require validation with patient and normal populations. Currently, the most common approaches have been to isolate mitochondria from biopsies, peripheral blood cells or develop fibroblast cell lines from patient samples. $3,14,16-19,22,46,47$ These methods have already been used to identify mitochondrial defects in diabetes and neurodegenerative diseases. ${ }^{3,4,18,48}$ The drawback of these approaches is that PBMC do not have high levels of mitochondria, biopsies are generally invasive and painful, and in the case of fibroblasts the passage of the cells may alter their bioenergetics. With the advent of extracellular flux analysis, it has become possible to apply these methods to much smaller numbers of cells that can be derived from small volumes of blood and perform multiple measurements on the same subject over time at a relatively low cost and without the requirement of surgery to obtain the sample. Future experiments will also determine how cellular bioenergetics can possibly act as a measure of the effectiveness of lifestyle interventions, such as exercise or diet, or the impact of aging on mitochondrial function.

Other applications are to use the specific cell types isolated from leukocytes as surrogate markers for bioenergetic disease in chronic systemic pathologies (eg, diabetes), as a direct investigation of contribution of the particular cell type to a disease (eg, platelets and thrombosis or endothelial cell 
a

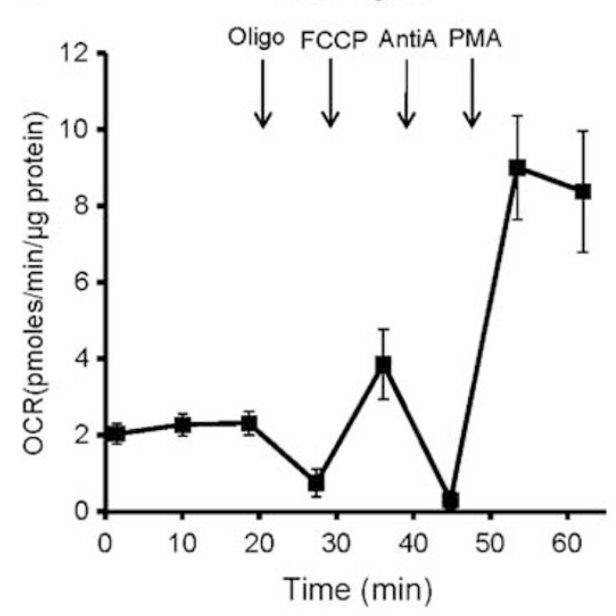

c

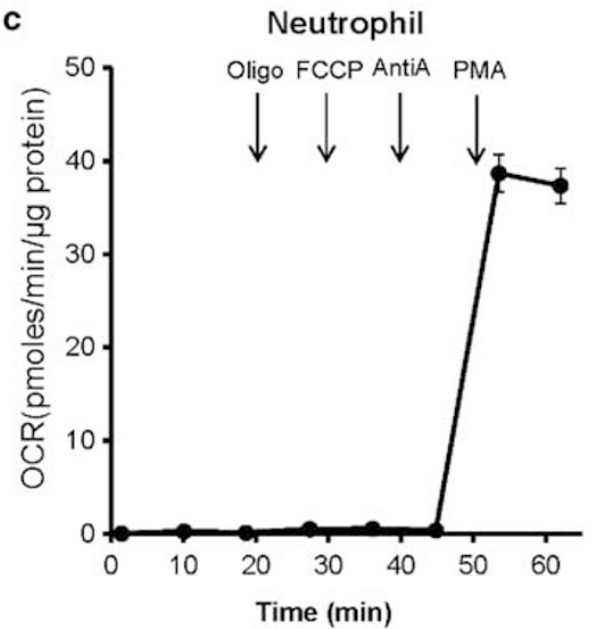

b PMA Response

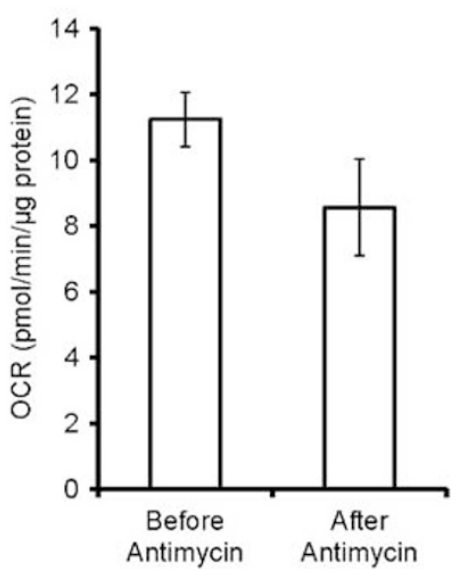

d PMA Response

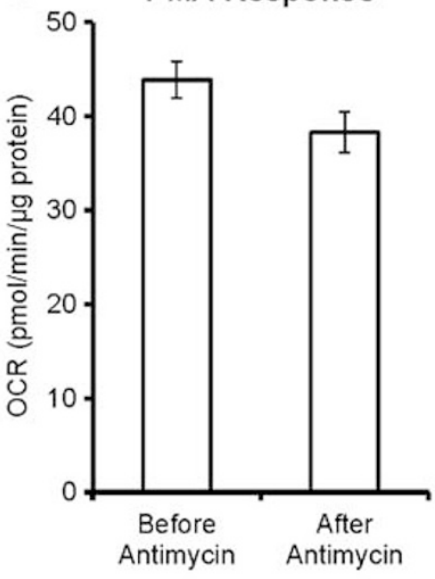

Figure 6 Activating the oxidative burst in monocytes and neutrophils following bioenergetic profiling. (a) The representative bioenergetic profile of CD14 ${ }^{+}$monocytes followed by an injection of $100 \mathrm{ng} / \mathrm{ml} \mathrm{PMA.} \mathrm{(b)} \mathrm{CD}_{14}{ }^{+}$monocyte PPR response to PMA from the same donor before and after $10 \mu \mathrm{M}$ antimycin A. (c) The representative bioenergetic profile of CD15 + neutrophils followed by an injection of $100 \mathrm{ng} / \mathrm{ml}$ PMA. (d) CD15 + neutrophil PPR response to PMA from the same donor before and after $10 \mu \mathrm{M}$ antimycin. The mean values \pm s.e.m. $n=3-5$ assay replicates per sample.

function) or as a means to monitor drug treatments. In the case of monocytes and neutrophils, there is an additional interest in assessing the ability of cells to generate superoxide through the NADPH oxidase and the extent to which a disease process primes the activation of this pathway. Importantly, there have been no studies characterizing the bioenergetic and ROS-generating profiles of platelets, neutrophils, lymphocytes, and monocytes from a single individual; the data and methodology provided in this report represent the first such studies, and can provide a basis for future assessments of these cell types and others.

Here, we have measured oxygen consumption and extracellular acidification in the cells that can be readily isolated from $10-20 \mathrm{ml}$ of human blood. The cell isolations can be completed within $4 \mathrm{~h}$ of sample collection, and by using selective isolation methods populations of monocytes, platelets, lymphocytes, and neutrophils can be prepared to $80 \%$ purity. The isolation procedure and attachment to the extracellular flux analysis does not result in detectable activation of the cells based upon morphological criteria (Supplementary Figure 1) although other more subtle changes cannot be ruled out.

In summary, we have shown that bioenergetic profiles can be obtained from a single blood sample for monocytes, platelets, and lymphocytes. Neutrophils have a negligible OCR that can be attributed to oxidative phosphorylation. These data are consistent with previous studies in which it was shown that neutrophils are primarily glycolytic, and have fewer mitochondria than other circulating leukocytes, which probably have a role in apoptosis. ${ }^{49,50}$ PMA stimulation can be used to measure changes in both OCR and PPR to assess oxidative burst in neutrophils and monocytes. The bioenergetic profiles of platelets, monocytes, and lymphocytes can be readily obtained and show interesting differences. The composition 
a

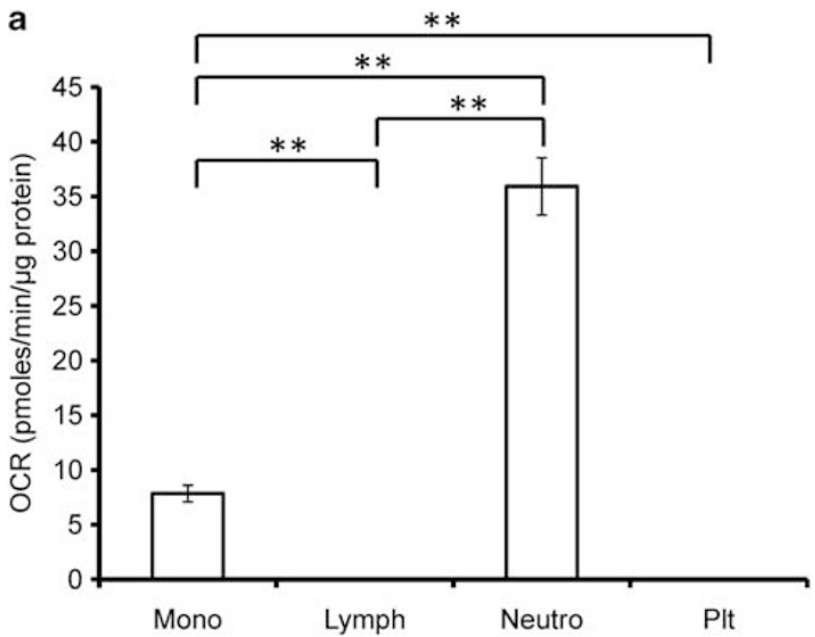

b

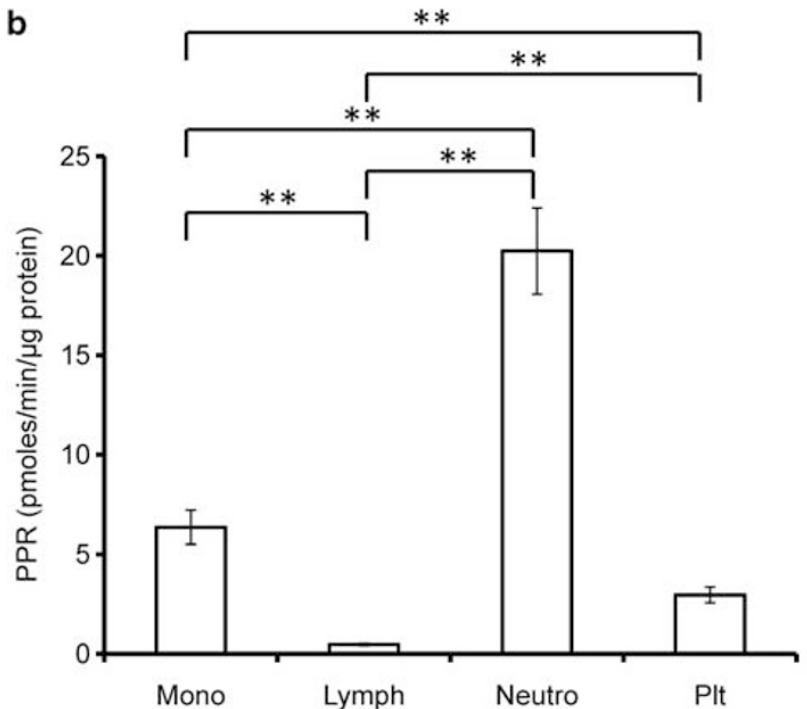

Figure 7 PMA response by cell type. (a) The PMA-stimulated OCR of monocytes, lymphocytes, neutrophils, and platelets are shown. (b) The PMA-stimulated PPR of monocytes, lymphocytes, neutrophils, and platelets are shown. Cumulative data from 6-8 healthy donors expressed as mean \pm s.e.m. ${ }^{*} P \leq 0.005$.

of mitochondria in monocytes, platelets, and lymphocytes appears to be different, although a full proteomics analysis is required to verify this. These data suggest that cells will be differentially sensitive to stressors that act at different segments of the respiratory chain and this should be considered when they are used for translational screens of bioenergetic defects. For example, because platelets are functioning at the near maximal energetic capacity under basal conditions, they may not be the best choice to measure defects in complexes I-IV. In contrast, monocytes and lymphocytes show a reserve bioenergetic capacity and may therefore be more appropriate for such analysis. Interestingly, because the levels of the components for electron transport complexes appear to be different between cell types it is likely that the control of respiration for each cell type is different, and suggests that exposure of these cells to the same mitochondrial stressor would likely give a different response. Clearly, much more extensive investigations are required to establish the range limits for mitochondrial function assays and oxidative burst analyses between donor, sex and age, and cell types. However, an appreciation of these unique features of cellular mitochondrial function represents a step forward in the understanding and interpretation of bioenergetic profiling in clinical samples.

Supplementary Information accompanies the paper on the Laboratory Investigation website (http://www.laboratoryinvestigation.org)

\section{ACKNOWLEDGEMENTS}

We thank Yaozu Ye, Taegyu Choi and Gloria A Benavides for their valuable technical assistance. This work was supported by T32HL07918 (PAK), P30DK056336 (BKC), DK079337 (VMD-U), HL103859 (SWB) and Diabetes Research Training Center Bioanalytical Redox Biology core (P60 DK079626).

\section{DISCLOSURE/CONFLICT OF INTEREST}

VMD-U is a member of the Seahorse Biosciences Scientific Advisory Board.

1. Srinivasan S, Yeh M, Danziger EC, et al. Glucose regulates monocyte adhesion through endothelial production of interleukin-8. Circ Res 2003:92:371-377.

2. Yamagishi Sl, Edelstein $\mathrm{D}, \mathrm{Du} \mathrm{XL}$, et al. Hyperglycemia potentiates collagen-induced platelet activation through mitochondrial superoxide overproduction. Diabetes 2001;50:1491-1494.

3. Avila C, Huang RJ, Stevens MV, et al. Platelet mitochondrial dysfunction is evident in type 2 diabetes in association with modifications of mitochondrial anti-oxidant stress proteins. Exp Clin Endocrinol Diabetes 2012;120:248-251.

4. Guo $\mathrm{X}, \mathrm{Wu} J, \mathrm{Du} \mathrm{J}$, et al. Platelets of type 2 diabetic patients are characterized by high ATP content and low mitochondrial membrane potential. Platelets 2009;20:588-593.

5. Mancuso M, Calsolaro V, Orsucci $D$, et al. Mitochondria, cognitive impairment, and Alzheimer's disease. Int J Alzheimers Dis 2009;2009, pii951548.

6. Shi P, Wei Y, Zhang J, et al. Mitochondrial dysfunction is a converging point of multiple pathological pathways in amyotrophic lateral sclerosis. J Alzheimer Dis 2010;20(Suppl 2):S311-S324.

7. Jin S. Autophagy, mitochondrial quality control, and oncogenesis. Autophagy 2006;2:80-84.

8. Lim KL, Ng XH, Grace LG, et al. Mitochondrial dynamics and Parkinson's disease: focus on parkin. Antioxid Redox Signal 2012; 16:935-949.

9. Hill BG, Benavides GA, Lancaster JR, et al. Integration of cellular bioenergetics with mitochondrial quality control and autophagy. Biol Chem 2012;393:1485-1512.

10. Perez-Pinzon MA, Stetler RA, Fiskum G. Novel mitochondrial targets for neuroprotection. J Cereb Blood Flow Metab 2012;32:1362-1376.

11. Chacko BK, Srivastava A, Johnson MS, et al. Mitochondria-targeted ubiquinone (MitoQ) decreases ethanol-dependent micro and macro hepatosteatosis. Hepatology 2011;54:153-163.

12. Chacko BK, Reily C, Srivastava A, et al. Prevention of diabetic nephropathy in Ins2(+/)(AkitaJ) mice by the mitochondria-targeted therapy MitoQ. Biochem J 2010;432:9-19.

13. Smith RA, Murphy MP. Animal and human studies with the mitochondria-targeted antioxidant MitoQ. Ann N Y Acad Sci 2010; 1201:96-103.

14. Fink $B D$, Herlein $J A, O$ 'Malley $Y$, et al. Endothelial cell and platelet bioenergetics: effect of glucose and nutrient composition. PLoS One 2012;7:e39430.

15. Cordero MD, De Miguel M, Moreno Fernandez AM, et al. Mitochondrial dysfunction and mitophagy activation in blood mononuclear cells of fibromyalgia patients: implications in the pathogenesis of the disease. Arthritis Res Ther 2010;12:R17.

16. Japiassu AM, Santiago AP, d'Avila JC, et al. Bioenergetic failure of human peripheral blood monocytes in patients with septic shock is 
mediated by reduced F1Fo adenosine-5'-triphosphate synthase activity. Crit Care Med 2011;39:1056-1063.

17. Widlansky ME, Wang J, Shenouda SM, et al. Altered mitochondrial membrane potential, mass, and morphology in the mononuclear cells of humans with type 2 diabetes. Transl Res 2010;156:15-25.

18. Mosconi L, de Leon M, Murray J, et al. Reduced mitochondria cytochrome oxidase activity in adult children of mothers with Alzheimer's disease. J Alzheimer Dis 2011;27:483-490.

19. Shi C, Guo K, Yew DT, et al. Effects of ageing and Alzheimer's disease on mitochondrial function of human platelets. Exp Gerontol 2008;43:589-594.

20. Lenaz G, Bovina C, Castelluccio C, et al. Mitochondrial complex I defects in aging. Mol Cell Biochem 1997;174:329-333.

21. D'Aurelio M, Merlo Pich M, Catani L, et al. Decreased Pasteur effect in platelets of aged individuals. Mech Ageing Dev 2001;122:823-833.

22. Satoh N, Shimatsu A, Himeno A, et al. Unbalanced M1/M2 phenotype of peripheral blood monocytes in obese diabetic patients: effect of pioglitazone. Diabetes Care 2010;33:e7.

23. Nagy G, Koncz A, Fernandez D, et al. Nitric oxide, mitochondrial hyperpolarization, and $\mathrm{T}$ cell activation. Free Radic Biol Med 2007;42:1625-1631.

24. van der Windt GJ, Everts B, Chang $\mathrm{CH}$, et al. Mitochondrial respiratory capacity is a critical regulator of CD8 $+\mathrm{T}$ cell memory development. Immunity 2012;36:68-78.

25. Alvehus $M$, Buren J, Sjostrom $M$, et al. The human visceral fat depot has a unique inflammatory profile. Obesity 2010;18:879-883.

26. Estep JM, Baranova $\mathrm{A}$, Hossain $\mathrm{N}$, et al. Expression of cytokine signaling genes in morbidly obese patients with non-alcoholic steatohepatitis and hepatic fibrosis. Obes Surg 2009;19:617-624.

27. Slattery ML, Fitzpatrick FA. Convergence of hormones, inflammation, and energy-related factors: a novel pathway of cancer etiology. Cancer Prev Res 2009;2:922-930.

28. Rosa JS, Heydari S, Oliver SR, et al. Inflammatory cytokine profiles during exercise in obese, diabetic, and healthy children. J Clin Res Pediatr Endocrinol 2011;3:115-121.

29. Onyango I, Khan S, Miller B, et al. Mitochondrial genomic contribution to mitochondrial dysfunction in Alzheimer's disease. J Alzheimer Dis 2006:9:183-193.

30. Hand WL, Hand DL, Vasquez Y. Increased polymorphonuclear leukocyte respiratory burst function in type 2 diabetes. Diabetes Res Clin Pract 2007;76:44-50

31. Omori K, Ohira T, Uchida Y, et al. Priming of neutrophil oxidative burst in diabetes requires preassembly of the NADPH oxidase. J Leukoc Biol 2008;84:292-301.

32. Zielinska-Przyjemska M, Olejnik A, Dobrowolska-Zachwieja A, et al. In vitro effects of beetroot juice and chips on oxidative metabolism and apoptosis in neutrophils from obese individuals. Phytother Res 2009;23:49-55.

33. Nieman DC, Nehlsen-Cannarella SL, Henson DA, et al. Immune response to exercise training and/or energy restriction in obese women. Med Sci Sports Exerc 1998;30:679-686.

34. Mossberg N, Andersen $\mathrm{O}$, Nordin $\mathrm{M}$, et al. Leukocyte oxygen radical production determines disease severity in the recurrent Guillain-Barre syndrome. J Inflamm (Lond) 2010;7:40.
35. Korzenik JR, Dieckgraefe BK. Is Crohn's disease an immunodeficiency? A hypothesis suggesting possible early events in the pathogenesis of Crohn's disease. Dig Dis Sci 2000;45:1121-1129.

36. Hultqvist M, Olofsson P, Holmberg J, et al. Enhanced autoimmunity, arthritis, and encephalomyelitis in mice with a reduced oxidative burst due to a mutation in the Ncf1 gene. Proc Natl Acad Sci USA 2004;101:12646-12651.

37. Agudelo-Florez P, Costa-Carvalho BT, Lopez JA, et al. Association of glucose-6-phosphate dehydrogenase deficiency and X-linked chronic granulomatous disease in a child with anemia and recurrent infections. Am J Hematol 2004;75:151-156.

38. Rosa-Borges A, Sampaio MG, Condino-Neto A, et al. [Glucose-6phosphate dehydrogenase deficiency with recurrent infections: case report]. J Pediatr (Rio J) 2001;77:331-336.

39. Winkelstein JA, Marino MC, Johnston Jr. RB, et al. Chronic granulomatous disease. Report on a national registry of 368 patients. Medicine (Baltimore) 2000;79:155-169.

40. Richardson MP, Ayliffe MJ, Helbert $M$, et al. A simple flow cytometry assay using dihydrorhodamine for the measurement of the neutrophil respiratory burst in whole blood: comparison with the quantitative nitrobluetetrazolium test. J Immunol Methods 1998; 219:187-193.

41. Matzner Y. Neutrophil function studies in clinical medicine. Transfus Med Rev 1987;1:171-181.

42. Walkowiak B, Kesy A, Michalec L. Microplate reader-a convenient tool in studies of blood coagulation. Thrombosis Res 1997;87:95-103.

43. Dranka BP, Benavides GA, Diers AR, et al. Assessing bioenergetic function in response to oxidative stress by metabolic profiling. Free Radic Biol Med 2011;51:1621-1635.

44. Chen Y, Junger WG. Measurement of oxidative burst in neutrophils. Methods Mol Biol 2012;844:115-124.

45. Hill BG, Dranka BP, Zou L, et al. Importance of the bioenergetic reserve capacity in response to cardiomyocyte stress induced by 4hydroxynonenal. Biochem J 2009;424:99-107.

46. Valentin-Vega YA, Maclean KH, Tait-Mulder J, et al. Mitochondrial dysfunction in ataxia-telangiectasia. Blood 2012;119:1490-1500.

47. Pavlides S, Vera I, Gandara R, et al. Warburg meets autophagy: cancerassociated fibroblasts accelerate tumor growth and metastasis via oxidative stress, mitophagy, and aerobic glycolysis. Antioxid Redox Signal 2012;16:1264-1284.

48. Blandini F, Nappi G, Greenamyre JT. Quantitative study of mitochondrial complex I in platelets of parkinsonian patients. Mov Disord 1998;13:11-15.

49. Borregaard N, Herlin T. Energy metabolism of human neutrophils during phagocytosis. J Clin Invest 1982;70:550-557.

50. Maianski NA, Maianski AN, Kuijpers TW, et al. Apoptosis of neutrophils. Acta Haematol 2004;111:56-66.

(c) (1) (5) (2) This work is licensed under the Creative Commons Attribution-NonCommercial-Share Alike

3.0 Unported License. To view a copy of this license, visit http://creativecommons.org/licenses/by-nc-sa/3.0/ 